\title{
25 Research Square \\ Refractive Error Induced by Intraocular Lens Tilt After Intrascleral Intraocular Lens Fixation
}

TERUAKI TOKUHISA ( $\nabla$ terrychicken1212@gmail.com )

Jikei University School of Medicine: Tokyo Jikeikai lka Daigaku https://orcid.org/0000-0002-0681246X

\section{TOMOYUKI WATANABE}

Jikei University School of Medicine: Tokyo Jikeikai Ika Daigaku

\section{AKIRA WATANABE}

Jikei University School of Medicine: Tokyo Jikeikai lka Daigaku

\section{TADASHI NAKANO}

Jikei University School of Medicine: Tokyo Jikeikai Ika Daigaku

\section{Research Article}

Keywords: Intrascleral fixation, IOL tilt, IOL decentration, CASIA2, refractive error, Zemax

Posted Date: March 19th, 2021

DOI: https://doi.org/10.21203/rs.3.rs-192757/v1

License: (c) (i) This work is licensed under a Creative Commons Attribution 4.0 International License.

Read Full License

Version of Record: A version of this preprint was published at International Ophthalmology on January 22nd, 2022. See the published version at https://doi.org/10.1007/s10792-021-02106-3. 


\section{Abstract}

Purpose To investigate the spherical shift of Intraocular lens (IOL) tilt after intrascleral fixation

Methods We retrospectively reviewed the medical records of patients who underwent flanged intrascleral IOL fixation with transconjunctival 25- or 27-gauge pars plana vitrectomy at the Department of Ophthalmology of Jikei University Hospital with a minimum follow-up duration of three months. Secondgeneration anterior segment optical coherence tomography (CASIA2; TOMEY) was used to obtain the tilt and decentration of intrasclerally fixated IOL and postoperative anterior chamber depth. We investigated the relationship of the refractive error with these parameters, axial length, and keratometry. In addition to the clinical investigation, we performed optical simulations using the Zemax optical design program for studying the spherical shift of the IOL tilt by means of the through-focus response and change of spherical equivalent power.

Results The study involved 72 eyes of 67 patients. The degree of IOL tilt was correlated with the amount of refractive error (Spearman's rank correlation coefficient $[C C]=-0.32 ; P=0.006$ ). In particular, a tilt angle greater than $10^{\circ}$ strongly influenced the refractive error. Postoperative anterior chamber depth also correlated with the refractive error $(C C=0.50 ; P<0.001)$. The refractive error did not correlate with decentration ( $\mathrm{CC}=-0.17 ; P=0.15)$, axial length $(\mathrm{CC}=-0.08 ; P=0.49)$, and keratometry $(\mathrm{CC}=-0.06 ; P$ $=0.64)$. Optical simulations using the Zemax optical design program also showed a myopic shift exponentially as the tilt becomes greater.

Conclusion An IOL tilt greater than $10^{\circ}$ induces refractive error.

\section{Introduction}

Intraocular lens (IOL) implantation to the eyes that lack or have insufficient capsular support has been challenging. Among the options in such cases are angle-supported anterior chamber IOL, iris-fixed IOL, and transscleral IOL suture [1,2]. However, each of these techniques presents several potential problems. Angle-supported anterior chamber IOL has a high rate of endothelial cell loss [3] and uveitis-glaucomahyphema syndrome [4]. Although the implantation of an iris-fixed IOL is an easy technique with low intrusiveness, problems of endothelial cell loss remain, and this technique is limited by the conditions of the iris and the anterior chamber depth (ACD) [5]. Transscleral IOL suture also presents problems, including suture-related complications such as endophthalmitis owing to suture exposure [6] and IOL dislocation induced by suture breakage [7].

Intrascleral fixation of both haptics in the ciliary sulcus was first introduced by Gabor et al [8]. This method has lower risks of suture-related complications, but this technique still requires suturing to close the scleral incision. Since then, various methods of intrascleral fixation have been reported [9-12]. The flanged intrascleral IOL fixation with double-needle technique reported by Yamane et al $[11,12]$ has become popular due to its minimal invasiveness and ease. The tilt and decentration of IOL after 
intrascleral fixation have been reported to be rather small [11], but some cases of severe tilting and refractive error occur simultaneously [13]. Thus, we hypothesized that IOL tilting causes refractive error.

In 1988, Uozato et al. [14] reported that the tilt and decentration of IOL cause spherical and astigmatic refractive changes using Purkinje reflections. Since then, however, some clinical studies have concluded that IOL tilt has no effect on refractive error $[11,15]$.

A second-generation anterior segment optical coherence tomography (CASIA2; TOMEY), which can measure postoperative tilt and decentration with good repeatability, was developed in 2015 [16]. This study aimed to evaluate the tilt, decentration, and ACD of scleral-fixated IOL using CASIA2 and to investigate the correlation between these parameters and refractive error.

\section{Methods}

This study was conducted with approval from the institutional review board and in accordance with the ethical standards of the Helsinki Declaration of 1975, as revised in 2000 and 2008. The study protocol was approved by the Institutional Review Board of the Jikei University School of Medicine (approval nomber:31-421[10003]), and all clinical procedures were conducted according to the principles of the Declaration of Helsinki.

The medical records of consecutive patients who underwent intrascleral IOL fixation between September 2017 and October 2020 at the Department of Ophthalmology of the Jikei University Hospital were retrospectively assessed. The study protocol was disclosed, and patients were given the opportunity to refuse to participate in the study. The inclusion criteria were as follows: 20 years of age or older, treatment with intrascleral IOL fixation for aphakia, a subluxated crystalline lens, or a dislocated IOL, and a postoperative follow-up duration of longer than three months. The exclusion criteria were as follows: patients with corneal disease (such as keratoconus or bullous keratopathy), those with incomplete operative or postoperative medical records.

The information collected were age, sex, preoperative lens status, axial length, pre- and postoperative refractive power measured by autorefractometry, the best corrected visual acuity, postoperative intraocular pressure (IOP), postoperative IOL status, ACD, IOL power, and intra- and postoperative complications. Postoperative hypotony and IOP elevation were defined as an IOP less than $6 \mathrm{mmHg}$ and greater than $25 \mathrm{mmHg}$, respectively. The difference between the predicted spherical equivalent calculated with the Barrett Universal II Formula and the actual objective refraction value at the final visit was determined to be the refractive error.

The characteristics of the 72 eyes of 67 patients included in this study are shown in the Supplementary Information. The study group included 59 men and 8 women. The mean age of the patients was $61.3 \pm$ 11.9 years (range, 37-86 years). The mean axial length was $25.28 \pm 1.56 \mathrm{~mm}$ (range, 21.89-29.02 mm). There were 6 cases of aphakia, 60 dislocated IOLs, and 6 subluxated crystalline lenses. The mean followup period was 14.3 months. 
Intrascleral IOL fixation was performed as described previously [12], with some modifications. A 25- or 27gauge pars plana vitrectomy was performed using a Constellation Vision System (Alcon Laboratories, Inc. Fort Worth, TX USA) under retrobulbar anesthesia. Phacoemulsification cataract extraction was performed for the subluxated crystalline lens. If the dislocated IOL was made of soft material, it was cut into two or three pieces and extruded from the 3.5-mm sclerocorneal incision. If the dislocated IOL was made of polymethyl methacrylate, it was removed through a $3 \times 3-\mathrm{mm}$ L-shaped scleral pocket incision.

A scleral tunnel incision was made with a trocar through the conjunctiva, sclera, and ciliary surfaces at the 2 o'clock, 10 o'clock, and temporal lower positions. A three-piece IOL (X-70, Santen Pharmaceutical, Co. Ltd. Japan) was inserted into the anterior chamber using an injector. An angled sclerotomy was made through the conjunctiva using a 30-gauge thin-wall needle (TSK Ultra Thin Wall Needle; Tochigi Seiko, Japan). The scleral penetrating point was $2.0 \mathrm{~mm}$ posterior from the limbus at the 2 o'clock position, aimed $20^{\circ}$ inward to the posterior chamber. The leading haptic was pushed into the lumen of the needle with forceps. A second sclerotomy was then made with a 30-gauge thin-wall needle at the 8 o'clock position. The same procedure was followed for the second haptic. Both haptics were pulled out, and the ends of the haptics were cauterized using an ophthalmic cautery device (Accu-Temp Cautery; BeaverVisitec International, Inc. USA) to make a flange. The flange of the haptics was pushed back and fixed into the scleral tunnel, and the IOL position was centered. A peripheral iridotomy was performed using the vitrectomy cutter after miosis to avoid iris capture of the IOL.

IOL Tilt, Decentration, and ACD Measurements

The tilt and decentration of the IOL and ACD were measured using CASIA2 at least three months after surgery. The CASIA2 uses a 1310-nm swept-source laser wavelength, producing 16 anterior segment optical coherence tomography (AS-OCT) images from 16 different angles and a three-dimensional analysis of the results. Furthermore, it automatically measures the tilt and decentration of the IOL relative to the corneal topographic axis, which connects the fixation point on the corneal topographer to the corneal vertex.

Analysis

The computer software SPSS (version 20; IBM, USA) was used for statistical analysis. The normality of all data samples was first checked by the Shapiro-Wilk test. Spearman's rank correlation coefficient was calculated to assess the relationships of the IOL tilt, IOL decentration, axial length, and average keratometry with refractive error since tilt and decentration data did not fulfill the criteria for normal distribution. Measurement data were expressed as the mean \pm standard deviation.

Optical simulations with ZEMAX

We performed optical simulations in addition to clinical research. A schematic eye [17] was modeled in the Zemax optical design program (Zemax, LLC, Washington, USA) to study the spherical shift of the IOL 
tilt by means of through-focus response (TFR) [18] and visual acuity (VA) estimated from the intersection point of modulation-transfer and modulation-threshold function. The modulation-threshold function is the reciprocal of the retinal-contrast sensitivity adapted from the results of Campbell and Green [19]. The values of every $5^{\circ}$ from 0 to $40^{\circ}$ were evaluated and subsequently interpolated to obtain smooth curves. 10 D, 20 D, and 27 D of spherical IOL X-70 (Santen Pharmaceutical, Co. Ltd., Japan) were used for this simulation. Anterior and posterior radii, central thickness, and a refractive index of X-70 were provided by Santen Pharmaceutical.

\section{Results}

The refractive error was $-0.86 \pm 1.52 \mathrm{D}$ (range, $-10.58-+0.47 \mathrm{D}$ ). The tilt, decentration, and ACD were $8.4 \pm 6.9^{\circ}$ (range, $0.6-35.8^{\circ}$ ), $0.46 \pm 0.29 \mathrm{~mm}$ (range, $0.04-1.74 \mathrm{~mm}$ ), and $4.38 \pm 0.40 \mathrm{~mm}$ (range, 3.39$5.22 \mathrm{~mm}$ ), respectively. The best corrected VA at postoperative month 3 was $-0.01 \pm 0.27 \log M A R$.

A correlation was found between the tilt and refractive error (Spearman's rank correlation coefficient [CC] $=-0.32 ; P=0.006$ ) (Fig. 1). No statistically significant correlation was found between the refractive error and decentration ( $\mathrm{CC}=-0.17 ; P=0.15)$ (Fig.2), axial length ( $\mathrm{CC}=-0.08 ; P=0.49)$, and average keratometry $(C C=-0.06 ; P=0.64)$. ACD was correlated with the refractive error $(C C=0.50 ; P<0.001)$ (Fig. 3).

Four cases showed an excessive IOL tilt of $35.8^{\circ}, 34.2^{\circ}, 28.8^{\circ}$, and $24.2^{\circ}$. The refractive errors of each were $-10.42 \mathrm{D},-4.7 \mathrm{D},-5.82 \mathrm{D}$, and $-2.03 \mathrm{D}$, respectively. In 61 cases with a tilt less than $10^{\circ}$, the mean tilt was $5.6 \pm 1.6^{\circ}$, and the mean refractive error was $-0.46 \pm 0.49 \mathrm{D}$. In 25 cases with a tilt less than $5^{\circ}$, the mean tilt was $3.0 \pm 1.2^{\circ}$, and the mean refractive error was $-0.29 \pm 0.52 \mathrm{D}$. Therefore, we determined that in cases in which the $\mathrm{IOL}$ tilt was less than $10^{\circ}$, the refractive error was relatively small. However, a tilt angle greater than $10^{\circ}$ significantly induced a refractive error.

Optical simulations with ZEMAX

Peak of TFR and VA showed myopic shift exponentially as tilt became greater (Fig. 4-6). These results were consistent with the results of our clinical research.

\section{Discussion}

Our results show that the averages of tilt, decentration, and ACD were $8.4 \pm 6.9^{\circ}, 0.46 \pm 0.29 \mathrm{~mm}$, and 4.38 $\pm 0.40 \mathrm{~mm}$, respectively. Refractive error was related to tilt $(C C=-0.32 ; P=0.006)$ and $\mathrm{ACD}(\mathrm{CC}=0.50 ; P<$ $0.001)$. There was no statistical correlation between decentration and refractive error $(C C=-0.17 ; P=0.15)$.

Several studies have reported evaluation of the tilt and decentration of the IOL to date. The Purkinje and Scheimpflug methods were reported in the 1980s [14,20,21]. AS-OCT and ultrasound biomicroscopy (UBM) have been used to demonstrate the position of implanted IOLs since the 2000s [22,23].

Measurements using AS-OCT for tilt and decentration have demonstrated higher repeatability than either 
the Purkinje method or the Scheimpflug method [24]. These studies have used the pupil center as a reference, but that may not be optimal because the pupillary axis is affected by the shape of the pupil and because surgical invasion may cause deformation of the pupil. In previous studies, the corneal vertex was reported to be a safer and more effective reference for the center of the excimer laser beam in refractive surgery for myopia compared with the center of the pupil [25]. Because the corneal vertex is not affected by the shape of the pupil, the corneal topographic axis is a better reference line to evaluate IOL tilt and decentration compared with the pupillary axis.

CASIA2 is a second-generation AS-OCT, with a 1310-nm swept-source laser wavelength, which was developed in 2015. CASIA2 measures the tilt of the IOL as a reference to the corneal topographic axis, produces 16 meridional scan images from 16 different angles, and defines the greatest angle between the corneal topographic axis and the IOL in the 16 images as the IOL tilt.

Kimura et al. [16] reported that tilt and decentration measurements of the in-the-bag IOL obtained using CASIA2 had high repeatability under both non-mydriatic and mydriatic conditions and are not affected by pupil shape. They report that the IOL tilt obtained by CASIA2 with detail is large compared with previous studies.

Some studies have referred to IOL tilt after intrascleral fixation. Kumar et al. reported that the mean angle between the IOL and the iris was $3.2 \pm 2.7^{\circ}$ on the horizontal axis and $2.9 \pm 2.6^{\circ}$ on the vertical axis, using straight line passing through the iris pigment epithelium on either side as the reference line [26]. In some studies, taking a straight line passing through the iridocorneal angles on either side as a reference line, the mean IOL tilt angle was evaluated 2.5 to $3.8^{\circ}$ on the horizontal axis and 2.2 to $2.8^{\circ}$ on the vertical axis $[11,12,16]$. Recently, Jujo et al. reported IOL tilt measured by CASIA2 after intrascleral fixation was $3.5 \pm$ $3.0^{\circ}[27]$.

In our series, the IOL tilt after intrascleral fixation was greater than that reported previously. There are two possible reasons for this. First, intrascleral fixation is a relatively new technique, and the introduction period of this operation in our hospital was included in the investigation period. Further, the learning curve of the surgical procedure should be considered. In second, Four cases with excessive IOL tilt affected the average tilt (Supplementary Information; cases $8,17,30$, and 46 ).

When IOL is positioned within the lens capsule, the IOL and capsular are supported by lens zonules, maximizing the chances of optimal surgical and refractive outcomes. Even if the posterior capsule is violated, the IOL may be placed in the ciliary sulcus, maintaining excellent visual outcomes [28]. However, transscleral-sutured IOL and scleral-fixated IOL may have extensive tilt and decentration, and ACD change owing to tilt and decentration as the support of the zonule is lacking.

Although the fact that longitudinal IOL displacement causes refractive error is well known [29,30], there have been few reports of correlation between tilt, decentration, and refractive error. In 1988, Uozato et al. [14] reported that IOL tilt causes spherical and astigmatic changes, and IOL power increases exponentially as tilt becomes larger. They concluded that tilt should be less than $5^{\circ}$. In 1990, Erickson et 
al. [30] simulated spherical and cylindrical refractive error induced by IOL tilt. It showed $3,7,11$, and $15^{\circ}$ tilting of $19 \mathrm{D} \mathrm{IOL}$ induce exponentially increasing myopic shift of $0.04 \mathrm{D}, 0.20 \mathrm{D}, 0.52 \mathrm{D}$, and $0.98 \mathrm{D}$, respectively. Since then, studies using either AS-OCT or UBM reported that IOL tilt has no effect on astigmatism and refractive error, unlike previous studies $[11,16]$. This discrepancy was possibly induced by the relatively small IOL tilt measured with AS-OCT and UBM. Kurimori et al [13]. reported two cases after intrascleral fixation showing severe tilting of $38.1^{\circ}$ and $25.3^{\circ}$ and strong myopic shift of $-4.50 \mathrm{D}$ and $-2.75 \mathrm{D}$, respectively. A second surgery to shorten the length of the both haptics by 2 to $3 \mathrm{~mm}$ reduced the excessive tilt and myopia-shifted refraction simultaneously in both cases.

In this study, almost no refractive error was detected in cases of a tilt less than $5^{\circ}$, and a small refractive error was found in cases with a tilt between $5^{\circ}$ and $10^{\circ}$. The refractive error increased exponentially as the tilt became greater. An extremely large tilt and strong refractive error were seen in four cases simultaneously (Fig. 1). These results were consistent with previous reports and optical simulation using Zemax. Therefore, we determined that $10^{\circ}$ of IOL tilt was an acceptable cutoff value when investigating the effect of IOL tilting on refractive error.

Snell's law describes the relationship between the angles of incidence and refraction when light passes through a boundary between two different isotropic media. As the IOL tilt effect angle of incidence from aqueous humor to the IOL, the angle of refraction from the IOL to the aqueous humor changes, leading to refractive error.

Although no correlation was found between decentration and refractive error in our study, Uozato et al. [14] noted this relationship was even smaller than the relationship between tilt and refractive error. Thus, a study with more cases would be desirable in the future. Although Axial length and keratometry were also reported to cause postoperative refractive error, no correlations were found in this clinical

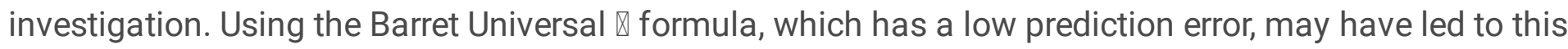
result [31].

Limitations of our study include its retrospective nature and a learning curve for scleral fixated IOL implantation.

In conclusion, intrasclerally fixated IOL tilt greater than $10^{\circ}$ strongly induces refractive error.

\section{Declarations}

\section{Funding}

No funding was received for this study

\section{Conflicts of interest}

Financial interests: Author T. Nakano has received Grant from CREWT Medical Systems, Kyowa Medical, Kuribara Medical Instruments, Kowa, Tomey, Otsuka Pharmaceutical, Senju Pharmaceutical, MSD, Pfizer, 
Alcon Japan, Santen Pharmaceutical, NIDEK, AMO Japan Bayer and IOL MEDICAL. Author T. Nakano has also received Personal Fees from Kowa, Otsuka Pharmaceutical, Senju Pharmaceutical, Santen Pharmaceutical, Nitto Medical and Nikon.

\section{Ethics approval}

This study was conducted with approval from the institutional review board and in accordance with the ethical standards of the Helsinki Declaration of 1975, as revised in 2000 and 2008. The study protocol was approved by the Institutional Review Board of the Jikei University School of Medicine (approval no.:31-421[10003) ), and all clinical procedures were conducted according to the principles of the Declaration of Helsinki.

Consent to participate (include appropriate statements)

The study protocol was disclosed, and patients were given the opportunity to refuse to participate in the study.

Consent to Publishment (include appropriate statements)

The study protocol was disclosed, and patients were given the opportunity to refuse to participate in the study.

\section{Availability of data and material (data transparency)}

Clinical data supporting the findings of this study are available within the article and its Supplementary Information.

Code availability (software application or custom code)

\section{References}

1)Bellucci $R$, zPucci V, Morselli S, Bonomi $L$ (1996) Secondary implantation of angle-supported anterior chamber and scleral-fixated posterior chamber intraocular lenses. J Cataract Refract Surg 22:247-252 https://doi.org/10.1016/s0886-3350(96)80227-6

2)Burkhard DH, Augustin AJ (2001) Lens implant selection with absence of capsular support. Curr Opin Ophthalmol 12:47-57. https://doi.org/10.1097/00055735-200102000-00009

3)Sawada T, Kimura W, Kimura T, Suga H, Ohte A, Yamanishi S, Ohara T (1998) Long-term follow-up of primary anterior chamber intraocular lens implantation. J Cataract Refract Surg 24:1515-1520. https://doi.org/10.1016/s0886-3350(98)80176-4

4) Ellingson FT (1978) The uveitis-glaucoma-hyphema syndrome associated with the Mark VIII anterior chamber lens implant. J Am Intraocul Implant Soc 4:50-53. https://doi.org/10.1016/s0146- 
5) De Silva SR, Arun K, Anandan M, Glover N, Patel CK, Rosen P (2011) Iris-claw intraocular lenses to correct aphakia in the absence of capsule support. J Cataract Refract Surg 37:1667- 1672. https://doi.org/10.1016/j.jcrs.2011.03.051

6) Heilskov T, Joondeph BC, Olsen KR, Blankenship GW (1989) Late endophthalmitis after transscleral fixation of a posterior chamber intraocular lens. Arch Ophthalmol107:1427.

https://doi.org/10.1001/archopht.1989.01070020501017

7) Price MO, Price FW Jr., Werner L, Berlie C, Mamalis N (2005) Late dislocation of scleral-sutured posterior chamber intraocular lenses. J Cataract Refract Surg 31:1320-1326.

https://doi.org/10.1016/j.jcrs.2004.12.060

8) Gabor SG, Pavlidis MM (2007) Sutureless intrascleral posterior chamber intraocular lens fixation. J Cataract Refract Surg 33:1851-1854. https://doi.org/10.1016/j.jcrs.2007.07.013

9) Agarwal A, Kumar DA, Jacob S, Baid C, Agarwal A, Srinivasan S (2008) Fibrine glue-assisted sutureless posterior chamber intraocular lens implantation in eyes with deficient posterior capsules $₫ \mathrm{~J}$ Cataract Refract Surg 34:1433-1438. https://doi.org/10.1016/j.jcrs.2008.04.040

10) Ohta $T$, Toshida $H$, Murakami A (2014) Simplified and safe method of sutureless intrascleral posterior chamber intraocular lens fixation: Y-fixation technique. J Cataract Refract Surg 40:2-7. https://doi.org/10.1016/j.jcrs.2013.11.003

11) Yamane $S$, Inoue $M$, Arakawa A, Kadonosono K (2014) Sutureless 27-gauge needle-guided intrascleral intraocular lens implantation with lamellar scleral dissection. Ophthalmology 121:61-66. https://doi.org/10.1016/j.ophtha.2013.08.043

12) Yamane S, Sato S, Maruyama-Inoue M, Kadonosono K (2017) Flanged Intrascleral Intraocular Lens Fixation with Double-Needle Technique. Ophthalmology124:1136-1142.

https://doi.org/10.1016/j.ophtha.2017.03.036

13) Kurimori HY, Inoue M, Hirakata A (2018) Adjustments of haptics length for tilted intraocular lens after intrascleral fixation. Am J Ophthalmol Case Rep 10:180-184. https://doi.org/10.1016/j.ajoc.2018.02.025

14) Uozato $H$, Okada $Y$, Hirai Saishin $M(1988)$ What are the tolerable limits of IOL tilt and decentration? Jpn Rev Clin Ophthalmol 82:2308-2311

15) Kemer Atik B, Altan C, Agca A, Kirmaci A, Yildirim Y, Genc S et al. (2020) The effect of intraocular lens tilt on visual outcomes in scleral-fixated intraocular lens implantation. Int Ophthalmol 40:717-724. https://doi.org/10.1007/s10792-019-01233-2 
16) Kimura S, Morizane Y, Shiode Y, Hirano M, Doi S, Toshima S et al. (2017) Assessment of tilt and decentration of crystalline lens and intraocular lens relative to the corneal topographic axis using anterior segment optical coherence tomography. PloS One12(9): e0184066.

https://doi.org/10.1371/journal.pone.0184066

17) Liou H-L, Brennan NA (1997) Anatomically accurate, finite model eye for optical modeling. J Opt Soc Am A 14:1684-1695. https://doi.org/10.1364/josaa.14.001684

18) Norrby $S$, Piers $P$, Campbell $C$, van der Mooren $M(1998)$ Determining the imaging quality of intraocular lenses. J. Cataract Refract. Surg24: 703-714. https://doi.org/10.1016/s0886-3350(98)802708

19) Campbell, F. W., Green, D. G. (1965) Optical and retinal factors affecting visual resolution. Journal of Physiology 181:576-593. https://doi.org/10.1113/jphysiol.1965.sp007784

20) Phillips P, Pérez-Emmanuelli J, Rosskothen HD, Koester CJ (1988) Measurement of intraocular lens decentration and tilt in vivo. J Cataract Refract Surg14: 129-135. https://doi.org/10.1016/s08863350(88)80086-5

21) Sasaki K, Sakamoto Y, Shibata T, Nakaizumi H, Emori Y (1989) Measurement of postoperative intraocular lens tilting and decentration using Scheimpflug images. J Cataract Refractive Surg 15:454457. https://doi.org/10.1016/s0886-3350(89)80071-9

22) Loya N, Lichter H, Barash D, Goldenberg-Cohen N, Strassmann E, Weinberger D (2001) Posterior chamber intra-ocular lens implantation after capsular tear: ultrasound bio-microscopy evaluation. $J$ Cataract Refract Surg 27:1423-7. https://doi.org/10.1016/s0886-3350(01)00786-6

23) Kumar DA, Agarwal A, Prakash G, Jacob S, Saravanan Y, Agarwal A (2011) Evaluation of intraocular lens tilt with anterior segment optical coherence tomography. Am J Ophthalmol; 151:406-12. https://doi.org/ 10.1016/j.ajo.2010.09.013

24) Ding X, Wang Q, Chang P, Li J, Savini G, Huang J et al. (2015) The Repeatability Assessment of Three Dimensional Capsule-Intraocular Lens Complex Measurements by Means of High-Speed SweptSource Optical Coherence Tomography. PLoS One 10: e0142556. https://doi.org/10.1371/journal.pone.0142556

25) Okamoto S, Kimura K, Funakura M, Ikeda N, Hiramatsu H, Bains HS. (2011) Comparison of wavefrontguided aspheric laser in situ keratomileusis for myopia: Coaxially sighted corneal-light-reflex versus lineof-sight centration J Cataract Refract Surg 37:1951-1960. https://doi.org/10.1016/j.jcrs.2011.05.040.

26冈Kumar DA, Agarwal A, Agarwal A, Chandrasekar R, Priyanka V (2015) Long-term assessment of tilt of glued intraocular lenses: an optical coherence tomography analysis 5 years after. Ophthalmology 122:4855. https://doi.org/10.1016/j.ophtha.2014.07.032 
27) Jujo T, Kogo J, Sasaki H, Sekine R, Sato K, Ebisutani S, Toyoda Y et al. (2021) 27-gauge trocarassisted sutureless intraocular lens fixation. BMC Ophthalmology 21:8 https://doi.org/10.1186/s12886020-01758-6

28) Dubey R, Birchall W, Grigg J. (2012) Improved refractive outcome for ciliary sulcus-implanted intraocular lenses. Ophthalmology 119:261-265. https://doi.org/10.1016/j.ophtha.2011.07.050

29) Lakshminarayanan V, Enoch JM, Raasch T, Crawford B, Nygaard RW (1986) Refractive changes induced by intraocular lens tilt and longitudinal displacement. Arch Ophthalmol 104:90-92. https://doi.org/10.1001/archopht.1986.01050130100030

30) Erickson P (1990) Effects of intraocular lens position errors on postoperative refractive error. J Cataract Refract Surg 16:305-311. https://doi.org/10.1016/s0886-3350(13)80699-2

31) Ronald B Melles, Jack T Holladay, William J Chang (2018) Accuracy of Intraocular Lens Calculation Formulas. Ophthalmology 125:169-178. https://doi.org/10.1016/j.ophtha.2017.08.027

\section{Figures}




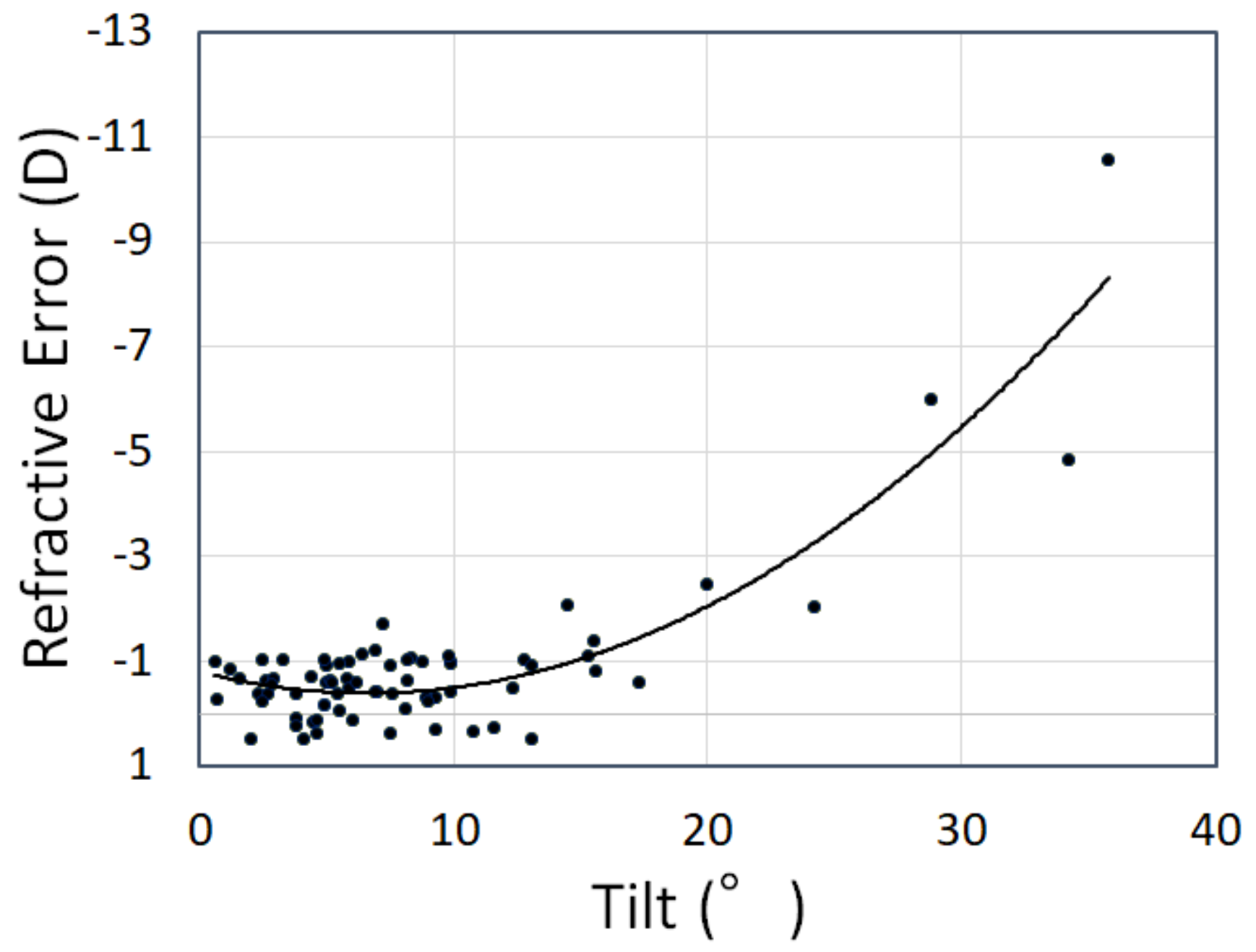

Figure 1

A significant correlation was found between the intraocular lens tilt and refractive error after intrascleral fixation (Spearman's rank correlation coefficient $=-0.32 ; \mathrm{P}=0.006$ ) 


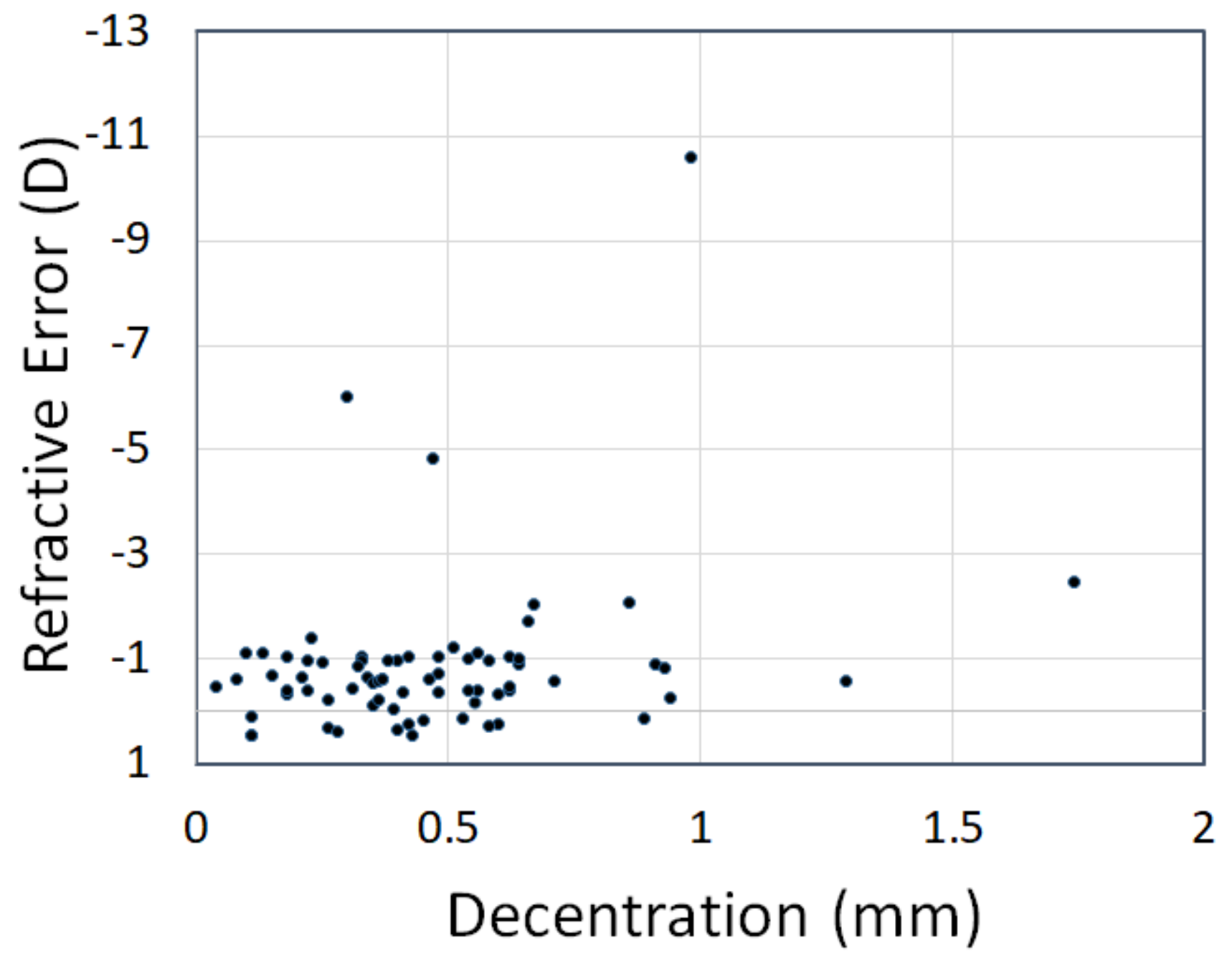

Figure 2

No statistical correlation was found between the intraocular lens decentration and refractive error after intrascleral fixation (Spearman's rank correlation coefficient $=-0.17 ; \mathrm{P}=0.15$ ) 


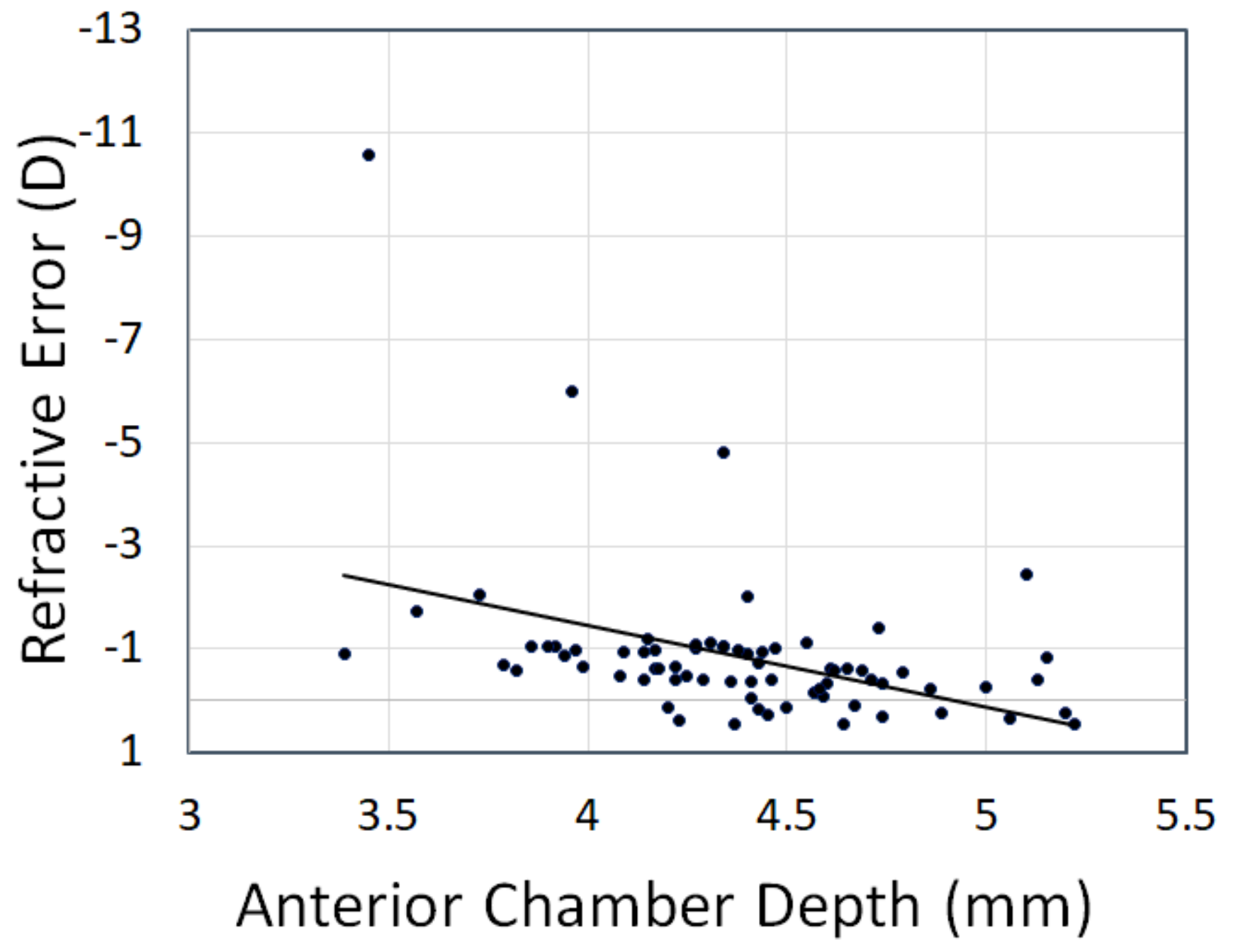

Figure 3

A significant correlation was found between the anterior chamber depth and refractive error after intrascleral fixation (Spearman's rank correlation coefficient $=0.50 ; \mathrm{P}<0.001$ ) 


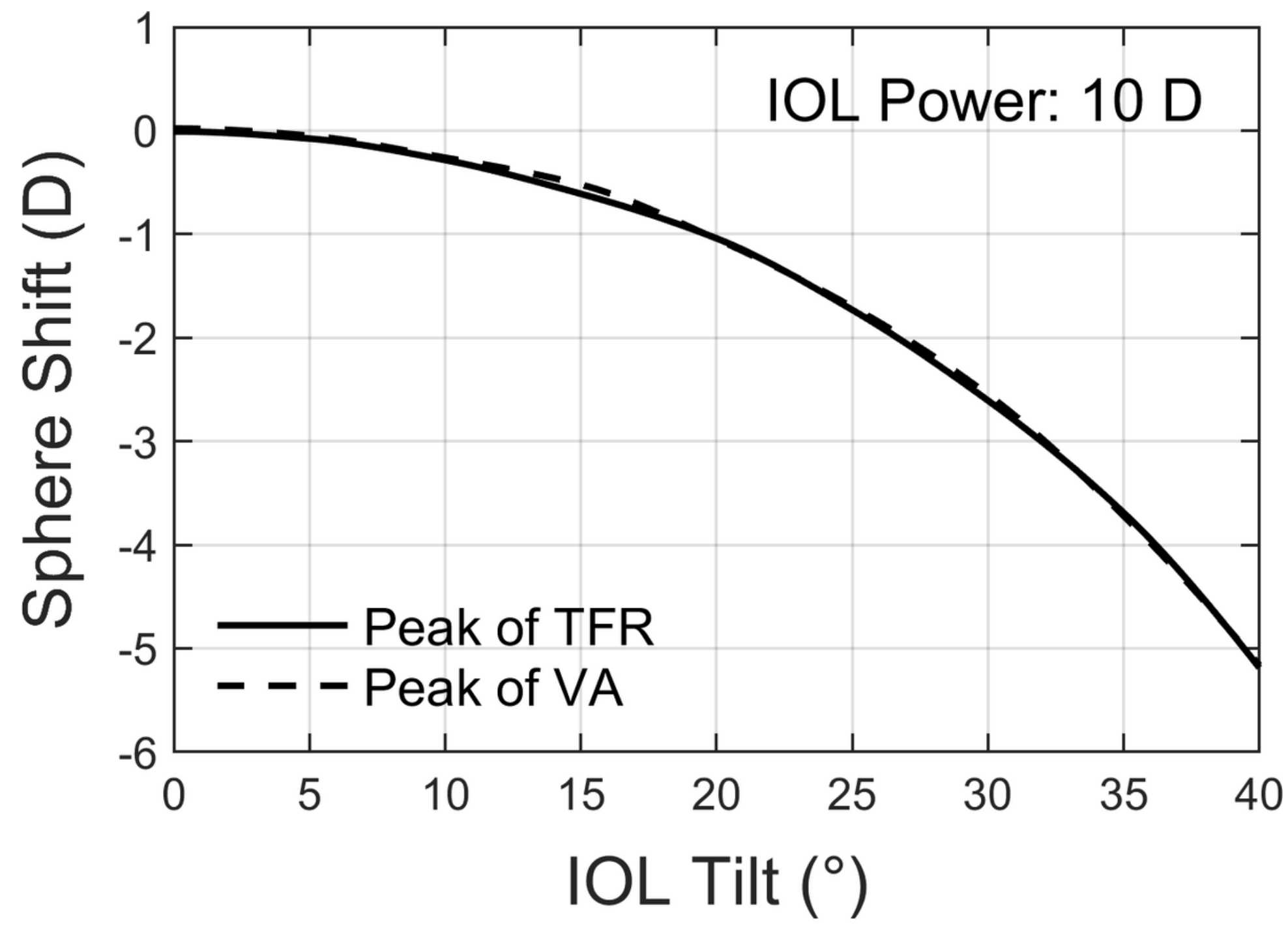

Figure 4

Sphere Shift Comparison of Peak of Through-Focus Response (TFR) and Visual Acuity (VA) (IOL power 10D) 


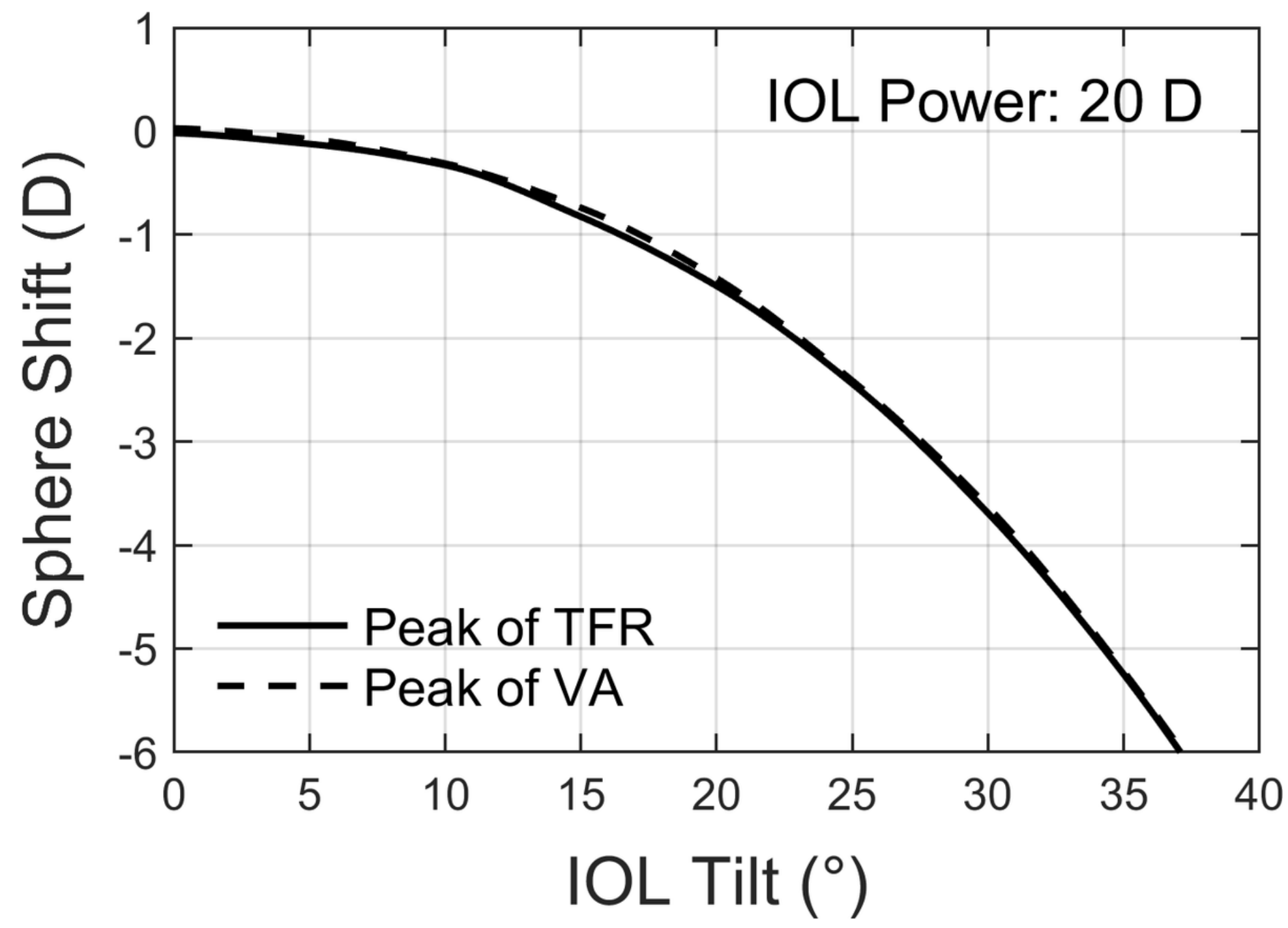

Figure 5

Sphere Shift Comparison of Peak of Through-Focus Response (TFR) and Visual Acuity (VA) (IOL power 20D) 


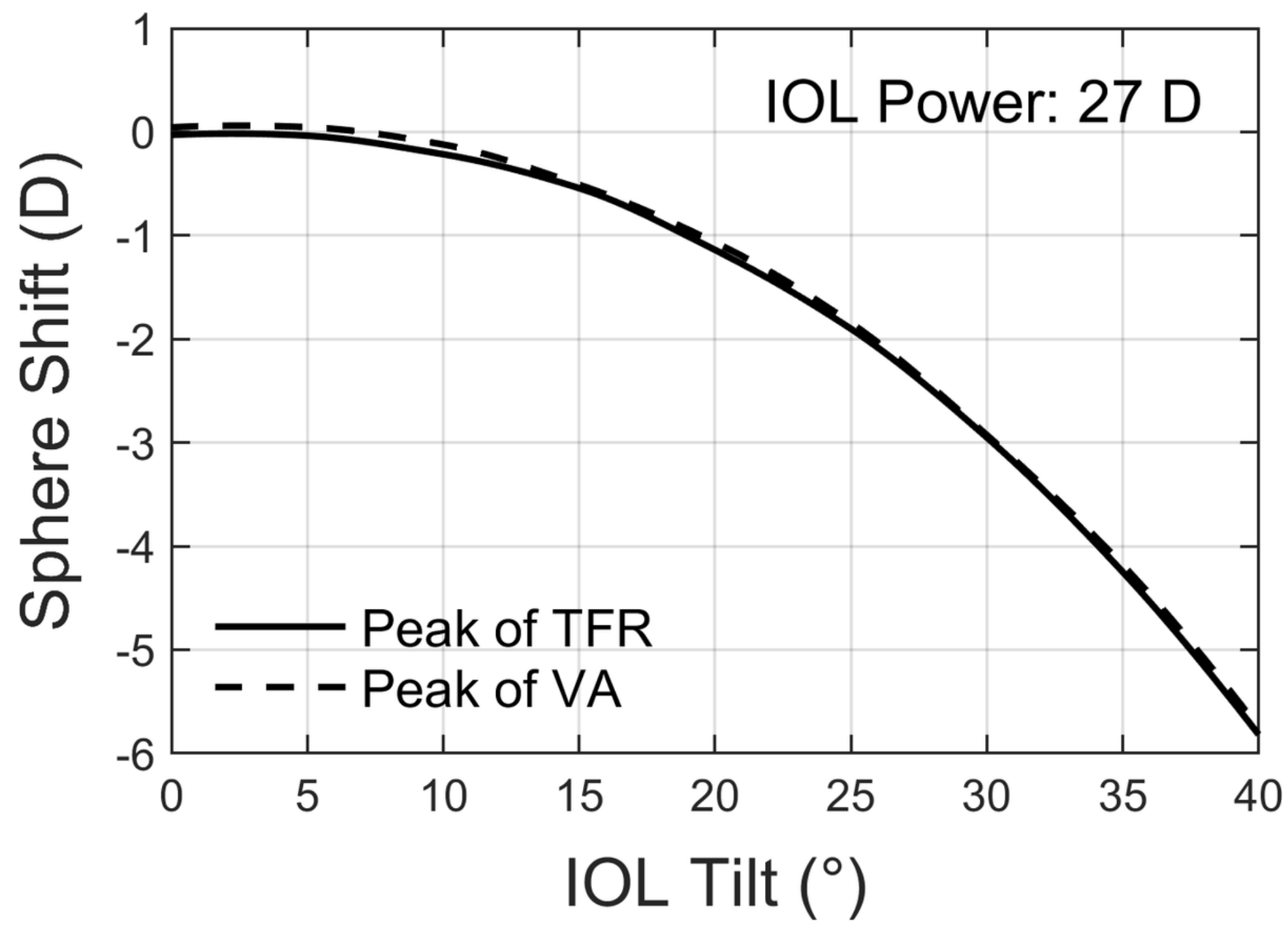

Figure 6

Sphere Shift Comparison of Peak of Through-Focus Response (TFR) and Visual Acuity (VA) (IOL power 27D)

\section{Supplementary Files}

This is a list of supplementary files associated with this preprint. Click to download.

- Supplementarylnformation.xlsx 